\title{
Mathematical Models of Therapeutical Actions Related to Tumour and Immune System Competition
}

\author{
Elena De Angelis ${ }^{(1)}$ and Pierre-Emmanuel Jabin ${ }^{(2)}$ \\ ${ }^{(1)}$ Dipartimento di Matematica, Politecnico di Torino \\ Corso Duca degli Abruzzi 24, 10129 Torino, Italy \\ email: elena.deangelis@polito.it \\ ${ }^{(2)}$ École Normale Supérieure \\ Département de Mathématiques et Applications, CNRS UMR 8553 \\ 45 rue d'Ulm, 75230 Paris Cedex 05, France \\ email: jabin@dma.ens.fr
}

\begin{abstract}
This paper deals with the qualitative analysis of a model related to the description of two medical therapies which have been intensively developed in recent years. In particular, we refer to the modeling of the actions applied by proteins, to activate the immune defense, and to the control of angiogenesis, to contrast the growth of tumour cells by preventing the feeding actions of endothelial cells. The therapeutical actions which are object of the modeling process developed in this paper have to be regarded as applied within the framework of the competition between the immune system and tumour cells. We prove the existence of solutions to the Cauchy problem related to the model. The efficiency of the therapies and the asymptotic behaviour in time of our solutions is also investigated.
\end{abstract}

Keywords: Vlasov kinetic theory, Cauchy problem, cell population, tumour-immune competition

\section{Introduction}

Methods of the mathematical kinetic theory have been applied in the last few years to model the competition between tumour and immune cells. The literature in the field and a critical analysis on the existing results and open problems can be found in the review papers [2] and [3].

Mathematical models are expected to describe the interactions and competition between tumours and the immune system. The evolution of the system may end up either with the blow-up of the host (with inhibition of the immune system), or with the suppression of the host due to the action of the immune system. The mathematical structure of the equations suitable to deal with the modeling of the above system have 
been developed and critically analyzed in [1], while motivations from scientists operating in the sciences of immunology in favor of development of methods of nonequilibrium statistical mechanics can be recovered, among others, in [12] and [13].

The mathematical methods are those typical in nonequilibrium statistical mechanics and generalized kinetic theory. The general idea, as documented in [4], consists in deriving an evolution equation for the first distribution function over the variable describing the microscopic internal state of the individuals. Generally, this variable may include position and velocity, but it can also refer to some additional specific microscopic features. Interactions between pairs have to be modeled taking into account not only mechanical rules but also modifications of the non-mechanical physical (internal) state.

Specifically we are interested in a development of the model proposed in [4], whose analytical properties have been studied in [5], toward the description of medical therapies which have been intensively developed in recent years. More precisely a model of competition between the immune system and tumour cells was proposed in [4], this model did not include the effect of any therapy. In [5], it was proved that there exists global solutions to this model and the possible asymptotic behaviours in time were detailed. The aim of this paper is first to present a general framework (in which the model of [4] fits) which is then used to build a new model. The difference with the previous model of [4] is that the effect of two different therapies is now taken into account. The last part of the paper studies the properties of the new model; Existence of global solutions is a consequence of the proof given in [5] and consequently we only state the result. However the analysis of the asymptotic behaviour in time is more complicated for the new model than what it was for the one of [4], thus requiring new ideas and new proofs which are presented.

Concerning the therapies, we refer to the modeling of the actions applied by proteins to activate the immune defense [15] and to the control of angiogenesis [9], [10], to contrast the growth of tumour cells by preventing the feeding actions of endothelial cells. All above therapeutical actions which are object of the modeling process developed in this paper have to be regarded as applied within the framework of the competition between the immune system and tumour cells. Referring to the literature on the immune competition, developed within medical sciences, the interested reader is addressed to the survey [6].

Another development of the model introduced in [4] is presented in [7], where the capacity of the body to repair cells damaged is taken into account. The idea is that for a long time range the body produces new cells to replace the dead ones in order to try to reach its normal healthy state.

After this introduction, the contents of this paper are organized in five more Sections:

- Section 2 deals with the derivation of a mathematical framework for the design of specific models suitable to describe the therapeutical actions indicated above. This means deriving a class of integro-differential equations to describe, by methods of the mathematical kinetic theory, the evolution over the biological state of the cells; 
- Section 3 deals with a short description of the two therapies we will consider: angiogenesis control and activation of the immune defense;

- Section 4 deals with some phenomenological assumptions about microscopic interactions, by which we obtain, within the general framework, the specific models object of our interest;

- Section 5 deals with the presentation of the analytical results related to the qualitative analysis of the solution to the initial value problem introduced in Section 4. This Section is also dedicated to the biological interpretation of the analytical results;

- Section 6 deals with the technical proofs of the results listed in Section 5.

\section{Mathematical framework towards modeling}

This section deals with the design of the general framework within which the specific models proposed in the next section will be developed. The objective is presented through three sequential steps. First we deal with the characterization of cell or particles population, then with the modeling of microscopic interaction, and finally with the derivation of a class of evolution equation. Specific models can be derived, as we shall see, by specializing the above mentioned microscopic interactions.

\subsection{Cell populations and statistical representation}

Consider a large system of cells or particles homogeneously distributed in space. Modeling the immune competition between tumour and immune cells under the medical action developed by particles which are artificially inserted into a vertebrate, needs the definition of the various populations which play the game.

In particular, we adopt the following assumptions:

Assumption 2.1 Cells and particles are homogeneously distributed in space. The system is constituted by the following populations: cells or particles of the aggressive host, immune cells, environmental cells, and particles of the therapeutical host (with possibly several different populations in this last class). Each population is labeled, respectively, by the indeces $i=1, \ldots, n$.

Assumption 2.2 Each cell is characterized by a certain state, a real variable $u \in$ $I=[0,+\infty)$ describing its main properties: progression for the host cells, activation for the immune cells, feeding ability for the environmental cells, and therapeutical ability for the particles of the therapeutical host.

Remark 2.1 The therapeutical ability needs to be specialized according to the specific medical action which is being modeled. In the cases which will be studied in the next section, we will consider two populations of particles with therapeutical effect and 
the therapeutical ability can hence be either the control of the activation ability of the immune system (for the first population), or the control of the feeding ability of the environmental cells (for the second population).

Assumption 2.3 The statistical description of the system is described by the number density functions

$$
N_{i}=N_{i}(t, u),
$$

which are such that $N_{i}(t, u) d u$ denotes the number of cells per unit volume whose state is, at time $t$, in the interval $[u, u+d u]$. Moreover, if $n_{0}$ is the number per unit volume of environmental cells at $t=0$,

$$
n_{0}=\int_{I} N_{3}(0, u) d u
$$

the description of the system can be given by the following distributions, normalized such as to have total density 1 at $t=0$ of environmental cells,

$$
f_{i}=f_{i}(t, u)=\frac{1}{n_{0}} N_{i}(t, u) .
$$

Other normalizations are of course possible. We opted for this one as the density of environmental cells represents in some sense the normal healthy state of the body.

Remark 2.2 If the distribution function $f_{i}$ is given, it is possible to compute, under suitable integrability properties, the size of the population still referred to $n_{0}$

$$
n_{i}(t)=\int_{I} f_{i}(t, u) d u,
$$

and first order moments such as the activation

$$
A_{i}(t)=A_{i}\left[f_{i}\right](t)=\int_{I} u f_{i}(t, u) d u,
$$

of each population.

\subsection{Modeling microscopic interactions}

This section deals with the modeling of microscopic interactions. Specifically we refer to the framework proposed in [4], which may be classified as mean field modeling, according to the fact that a test cell feels the presence and interacts with the surrounding field cells localized in a suitable volume around the test cell.

Assumption 2.4 Interactions are homogeneously distributed in space and can be divided into three types of encounters: mass conservative interactions, which modify the state of the pair, but not the size of the population, proliferative and destructive interactions, which produce proliferation or destruction of the interacting subjects, and population shifting interactions which generate individuals into a third population out of interactions within individuals of two different populations. 
Assumption 2.5 Conservative encounters are interactions modeled by the term $\mathcal{P}_{i k}=$ $\mathcal{P}_{i k}\left(u, u^{*}\right)$ which defines the action on the cell of the $i$-th population with microscopic state $u$ due to the cell, with state $u^{*}$, of the $k$-th population, so that the resultant action is

$$
\mathcal{F}_{i k}[\mathbf{f}](t, u)=\int_{I} \mathcal{P}_{i k}\left(u, u^{*}\right) f_{k}\left(t, u^{*}\right) d u^{*},
$$

where $\mathbf{f}=\left\{f_{1}, \ldots, f_{n}\right\}$.

Additional conditions should be considered on the term $\mathcal{P}_{i k}$ for $u=0$ to guarantee the conservation property of the term (2.3) on the interval $[0, \infty[$. We will see, in Section 4, that under the phenomenological assumptions we consider, such conditions are automatically satisfied.

Assumption 2.6 The term describing proliferation and/or destruction phenomena, within the same population, in the state $u$ due to pair interactions between cells of the $i$-th population with microscopic state $u^{*}$ with cells of the $k$-th population, with microscopic state $u^{* *}$, is modeled by the source/sink term:

$$
\mathcal{S}_{i k}[\mathbf{f}](t, u)=\int_{I} \int_{I} \sigma_{i k}\left(u^{*}, u^{* *} ; u\right) f_{i}\left(t, u^{*}\right) f_{k}\left(t, u^{* *}\right) d u^{*} d u^{* *},
$$

where $\sigma_{i k}$ is a suitable proliferation-destruction function.

Assumption 2.7 The term describing proliferation and/or destruction phenomena, within the $i$-th population, in the state $u$ related to pair interactions between cells of the $j$-th population with microscopic state $u^{*}$ due to the subject of the $k$-th population, with microscopic state $u^{* *}$ is given by:

$$
\mathcal{Q}_{j k}^{(i)}[\mathbf{f}](t, u)=\int_{I} \int_{I} \psi_{j k}^{(i)}\left(u^{*}, u^{* *} ; u\right) f_{j}\left(t, u^{*}\right) f_{k}\left(t, u^{* *}\right) d u^{*} d u^{* *},
$$

where $\psi_{j k}^{(i)}$ is a suitable proliferation-destruction function.

Assumption 2.8 The above framework refers to the evolution in absence of source/sink terms. This means that cells are contained in a vessel and the system is closed. Tumour cells can then replicate exploiting the existing environmental cells. On the other hand, the above mentioned supply or consumption can be modeled for the $i$-th population by suitable source/sink terms $\mathcal{I}_{i}(t, u)$

\subsection{Evolution equations}

The mathematical model consists in an evolution equation for the distribution functions $f_{i}$ corresponding to the above mentioned cell populations. The mathematical structure of the model proposed in Section 5 of [4] is as follows:

$$
\frac{\partial}{\partial t} f_{i}(t, u)+\mathcal{F}_{i}[\mathbf{f}](t, u)=\mathcal{S}_{i}[\mathbf{f}](t, u)+\mathcal{Q}_{i}[\mathbf{f}](t, u)+\mathcal{I}_{i}(t, u),
$$


where, according to the framework for microscopic modeling described in Eqs. (2.3), (2.4), (2.5), we have:

$$
\begin{gathered}
\mathcal{F}_{i}[\mathbf{f}](t, u)=\frac{\partial}{\partial u}\left[f_{i}(t, u) \sum_{k=1}^{n} \mathcal{F}_{i k}[\mathbf{f}](t, u)\right], \\
\mathcal{S}_{i}[\mathbf{f}](t, u)=\sum_{k=1}^{n} \mathcal{S}_{i k}[\mathbf{f}](t, u)
\end{gathered}
$$

and

$$
\mathcal{Q}_{i}[\mathbf{f}](t, u)=\sum_{j=1}^{n} \sum_{k=1}^{n} \mathcal{Q}_{j k}^{(i)}[\mathbf{f}](t, u) .
$$

This general framework can generate specific models after a detailed modeling of microscopic cell interactions, as it will be shown in Section 4.

\section{On the control of angiogenesis and immune activation}

The framework described in Section 2 can be exploited to derive specific models after having properly modeled all microscopic interactions among the various subjects playing the game. Of course the specific therapeutical actions which are applied has to be properly specified.

A large variety of therapeutical actions are known in the field of medicine: a brief account is given in this section with reference to the following two specific actions (we refer the interested reader to [3] for more details)

- Modeling of the actions applied by proteins to activate the immune defense, e.g. [11] or [15], thus preventing the ability of tumour cells to inhibit immune cells.

- Control of angiogenesis phenomena, that is the formation of new blood vessel from pre-existing vasculature, e.g. [9] and [10], thus preventing tumour growth by limiting the feeding ability from blood vessels.

There is an experimental evidence that immunotherapy have the potential to treat many tumour types, see [8]. The immunotherapy approach consists in the activation of specific tumour antigen combined with incorporation of an immunological adjuvant into a vaccine regime. In detail, cancer vaccines involve the induction of an active immune response that may lead to the subsequent destruction of tumour tissue. On the other hand, as reported in [16], an adoptive cancer immunotherapy involves the use of tumour-killing lymphocytes and lymphokines engaging in a search and destroy anticancer activity. Following [11], tumour vaccine and cytokine therapy are two methods of promoting an anticancer immune response and these techniques are highly effective when combined. In cancer prevention using cancer vaccines the target is not the tumour mass but the potential risk of cancer (the so-called primary prevention), a preneoplastic lesion (the so-called secondary prevention) or a small number of isolated 
neoplastic cells remaining after a temporarily successful therapeutical treatment (the tertiary prevention). Moreover, vaccination after the removal of a tumour mass can stop the formation of minimal residual disease or metastatic diffusion.

Referring to the second therapy, e.g. the control of angiogenesis phenomena, tumour progression and growth cannot occur without angiogenesis, which supplies the necessary oxygen and nutrients to the growing tumour. Various angiogenesis inhibitors have been developed to target endothelial cells and stop the process. Referring to [10], a new class of drugs is represented by different type of angiogenic inhibitors and they are extremely important in cases for which the general rules involving conventional chemotherapy might not apply. Inhibitors like angiostatin prevent vascular endothelial cells from proliferating and migrating, while indirect angiogenesis inhibitors can prevent the expression of the activity of one of the tumour proteins which drive the angiogenic switch. Another feature of the angiogenesis process is the evident abnormal vasculature as a hallmark of solid tumour, see [14], and the normalization of this abnormal vasculature can facilitate drug delivery to tumours and it represents an other important goal in the antiangiogenic therapy.

\section{Modeling the immune competition and the therapeuti- cal actions}

The general principles followed toward modeling are precisely the same we have seen in Section 2. Interactions with cells of the other populations modify the biological state and may generate proliferation and/or destruction phenomena. In detail, referring to interactions between host, immune and endothelial cells, we shall essentially extend the model proposed in [4] and [5]. For $i=1,2,3$ we will indicate, respectively, the aggressive host, the immune system and the environmental cells. Concerning the angiogenesis control and the activation of the immune system, let the related population of particles be denoted respectively by the subscript $i=4$ and $i=5$. The assumptions which define the microscopic interactions can be stated as follows.

Consider first conservative encounters described by Eq. (2.3):

Assumption 4.1 The progression of neoplastic cells is not modified by interactions with other cells of the same type. On the other hand, it is weakened by interaction with immune cells (linearly depending on their activation state) and it is increased by interactions with environmental cells (linearly depending on their feeding ability). The effect increases with increasing values of the progression. Moreover, the progression of the aggressive host is not modified by interactions with both particles of the antiangiogenesis and of the immune activation therapeutical actions:

$$
\mathcal{P}_{11}=0, \quad \mathcal{P}_{12}\left(u, u^{*}\right)=-\alpha_{12} u u^{*}, \quad \mathcal{P}_{13}\left(u, u^{*}\right)=\alpha_{13} u u^{*}, \quad \mathcal{P}_{14}=\mathcal{P}_{15}=0
$$

Assumption 4.2 The defense ability of immune cells is weakened by interactions with tumour cells (linearly depending on their activation state) due to their ability to inhibit the immune system. On the other hand, it is not modified by interactions with other 
cells of the same type, with environmental cells and with the angiogenic therapeutical host. Moreover, it is increased by interaction with the immune activation therapeutical host and the effect is linearly depending on their activation state:

$$
\mathcal{P}_{21}\left(u, u^{*}\right)=-\alpha_{21} u u^{*}, \quad \mathcal{P}_{22}=\mathcal{P}_{23}=\mathcal{P}_{24}=0, \quad \mathcal{P}_{25}\left(u, u^{*}\right)=\alpha_{25} u u^{*} .
$$

Assumption 4.3 The feeding ability of the endothelial cells is weakened by interaction with tumour cells linearly depending on their activation state. On the other hand, it is not modified by interactions with immune cells and with other cells of the same type. Moreover, it is weakened by interaction with the therapeutical host and we assume that this action depends linearly on their activation state. Finally it is not modified by interactions with the immune activation therapeutical host:

$$
\mathcal{P}_{31}\left(u, u^{*}\right)=-\alpha_{31} u u^{*}, \quad \mathcal{P}_{32} \mathcal{P}_{33}=0, \quad \mathcal{P}_{34}\left(u, u^{*}\right)=-\alpha_{34} u u^{*}, \quad \mathcal{P}_{35}=0 .
$$

Assumption 4.4 The therapeutical ability of the angiogenic therapeutical host is not modified by interactions with all the other populations and with cells of the same type:

$$
\mathcal{P}_{41}=\mathcal{P}_{42}=\mathcal{P}_{43}=\mathcal{P}_{44}=\mathcal{P}_{45}=0 .
$$

Assumption 4.5 The therapeutical ability of the immune activation host is not modified by interactions with all the other populations and with cells of the same type:

$$
\mathcal{P}_{51}=\mathcal{P}_{52}=\mathcal{P}_{53}=\mathcal{P}_{54} \mathcal{P}_{55}=0
$$

Consider now the nonconservative encounters described by Eq. (2.4). A simple modeling can be based on the assumption that the terms $\sigma_{i j}$ are delta functions over the state $u^{*}$ of the interacting test cell:

$$
\sigma_{i j}\left(u^{*}, u^{* *} ; u\right)=s_{i j}\left(u^{*}, u^{* *}\right) \delta\left(u-u^{*}\right),
$$

for all $i, j=1, \ldots, 5$.

Assumption 4.6 No proliferation of neoplastic cells occurs due to interactions with other cells of the same type. On the other hand, interactions with immune cells generate a destruction linearly depending on their activation state, while interactions with environmental cells generate a proliferation depending on their feeding ability and the progression of tumour cells. Moreover, no proliferation arises due to the interactions with the therapeutical hosts:

$$
s_{11}=0, \quad s_{12}\left(u^{*}, u^{* *}\right)=-\beta_{12} u^{* *}, \quad s_{13}\left(u^{*} u^{* *}\right)=\beta_{13} u^{*} u^{* *}, \quad s_{14}=s_{15}=0 .
$$

Assumption 4.7 Proliferation of immune cells occurs due to interactions with tumour cells, linearly depending on their defense ability and on the activation state of tumour cells. On the other hand, no proliferation of immune cells occurs due to interactions with other cells of the same type, with environmental cells and with cells of the therapeutical hosts:

$$
s_{21}\left(u^{*}, u^{* *}\right)=\beta_{21} u^{*} u^{* *}, \quad s_{22}=s_{23}=s_{24}=s_{25}=0 .
$$


Assumption 4.8 A destruction of the environmental cells occurs due to interactions with tumour cells, linearly depending on the activation state of tumour cells. On the other hand, no proliferation of environmental cells occurs due to interactions with immune cells, with other cells of the same type and with cells of the therapeutical hosts:

$$
s_{31}\left(u^{*}, u^{* *}\right)=-\beta_{31} u^{* *}, \quad s_{32}=s_{33}=s_{34}=s_{35}=0 .
$$

Assumption 4.9 No proliferation of cells of the angiogenic therapeutical host occurs due to interactions with tumour cells and with immune cells. On the other hand, a destruction occurs due to interactions with environmental cells, linearly depending on the activation state of environmental cells. No proliferation occurs due to interactions with cells of the same type and with cells of the immune activation therapeutical host:

$$
s_{41}=s_{42}=0, \quad s_{43}\left(u^{*}, u^{* *}\right)=-\beta_{43} u^{* *}, \quad s_{44}=s_{45}=0 .
$$

Assumption 4.10 No proliferation of cells of the immune activation therapeutical host occurs due to interactions with tumour cells. On the other hand, a destruction occurs due to interactions with immune cells, linearly depending on the activation state of the cells of the host. Moreover, no proliferation occurs due to interactions with the immune cells, with cells of the angiogenic therapeutical host and with other cells of the same type:

$$
s_{51}=0, \quad s_{52}=-\beta_{52} u^{* *}, \quad s_{53}=s_{54}=s_{55}=0 .
$$

Assumption 4.11 For all the actions we consider, the terms $\mathcal{Q}_{j k}^{(i)}$ in Eq. (2.5) are equal to zero. This means that the possibility of shift between populations is not relevant.

Assumption 4.12 No source terms are considered in the evolution equations for all the distribution functions $f_{i}$, expressing the absence of source/sink terms.

Based on the above modeling of cell interactions, we are now able to derive the evolution equations (2.6) for each $f_{i}$ :

$$
\begin{aligned}
\frac{\partial f_{1}}{\partial t}(t, u) & =\frac{\partial}{\partial u}\left[u f_{1}(t, u)\left(\alpha_{12} A_{2}\left[f_{2}\right](t)-\alpha_{13} A_{3}\left[f_{3}\right](t)\right)\right] \\
& +f_{1}(t, u)\left(-\beta_{12} A_{2}\left[f_{2}\right](t)+\beta_{13} u A_{3}\left[f_{3}\right](t)\right), \\
\frac{\partial f_{2}}{\partial t}(t, u) & =\frac{\partial}{\partial u}\left[u f_{2}(t, u)\left(\alpha_{21} A_{1}\left[f_{1}\right](t)-\alpha_{25} A_{5}\left[f_{5}\right](t)\right)\right] \\
& +\beta_{21} u f_{2}(t, u) A_{1}\left[f_{1}\right](t), \\
\frac{\partial f_{3}}{\partial t}(t, u) & =\frac{\partial}{\partial u}\left[u f_{3}(t, u)\left(\alpha_{31} A_{1}\left[f_{1}\right](t)+\alpha_{34} A_{4}\left[f_{4}\right](t)\right)\right] \\
& -\beta_{31} f_{3}(t, u) A_{1}\left[f_{1}\right](t),
\end{aligned}
$$




$$
\begin{aligned}
& \frac{\partial f_{4}}{\partial t}(t, u)=-\beta_{43} f_{4}(t, u) A_{3}\left[f_{3}\right](t), \\
& \frac{\partial f_{5}}{\partial t}(t, u)=-\beta_{52} f_{5}(t, u) A_{2}\left[f_{2}\right](t),
\end{aligned}
$$

for all $t, u \in \mathbb{R}^{+}$, where the activations $A_{i}\left[f_{i}\right]$ are defined in Eq. (2.2).

The Cauchy problem for the system (4.1) - (4.5) is defined given the initial conditions

$$
f_{i}(t=0, u)=f_{i}^{0}(u), \quad \forall i=1, \ldots, 5,
$$

for all $u \in \mathbb{R}^{+}$.

All the parameters $\alpha$ and $\beta$, which appear in the above Assumptions, have to be regarded as positive, small with respect to one, constants, to be identified by suitable experiments.

\section{Analytic Results and Biological Interpretation}

In the first part of this section we present the analytical results related to the qualitative analysis of the solution to the Cauchy problem (4.1) - (4.6) and we refer to the next Section 6 for the technical proofs.

The following theorem states the existence of the solutions of the Cauchy problem (4.1) - 4.6.

Theorem 5.1 Assume that the initial conditions $f_{i}^{0}$, for $i=1, \ldots, 5$, satisfy respectively the following assumptions

$$
\begin{gathered}
\int_{0}^{\infty} e^{\lambda u} f_{1}^{0}(u) d u<\infty, \quad \forall \lambda>0, \\
\int_{0}^{\infty}(1+u) e^{\lambda u} f_{2}^{0}(u) d u<+\infty, \quad \forall \lambda>0, \\
\int_{0}^{\infty}(1+u) f_{i}^{0}(u) d u<\infty, \quad \forall i=3,4,5 .
\end{gathered}
$$

Then there exists at least one solution $\left(f_{1}, f_{2}, f_{3}, f_{4}, f_{5}\right) \in C\left([0, \infty), L^{1}((1+u) d u)\right)$ to the initial value problem (4.1)-(4.6) (in the distributional sense) which satisfy

$$
\begin{aligned}
& \int_{0}^{\infty} e^{\lambda u} f_{1}(t, u) d u \in L^{\infty}([0, T]), \quad \forall \lambda, T>0, \\
& \int_{0}^{\infty}(1+u) e^{\lambda u} f_{2}(t, u) d u \in L^{\infty}([0, T]), \quad \forall \lambda, T>0, \\
& \int_{0}^{\infty}(1+u) f_{i}(t, u) d u \leq \int_{0}^{\infty}(1+u) f_{i}^{0}(u) d u, \quad \forall i=3,4,5 .
\end{aligned}
$$


Notice that to obtain the existence of solutions, we would only require to ask that $\int e^{\lambda u} f_{2}^{0}(u) d u$ be finite for all $\lambda>0$. The stronger hypothesis (5.2) is however useful in what follows, which concerns the asymptotic behaviour in time.

We also point out that the equation for $f_{4}$ and $f_{5}$ are not really necessary. Indeed the only interesting quantities are $A_{4}$ and $A_{5}$ and the relative equations, together with the equation for $A_{3}$, are listed in the following lemma.

Lemma 5.1 For the solutions to the initial value problem (4.1)-(4.6) given by Theorem 5.1 we have

$$
\begin{aligned}
\frac{d}{d t} A_{3}(t)=-\left[\left(\alpha_{31}\right.\right. & \left.\left.+\beta_{31}\right) A_{1}(t)+\alpha_{34} A_{4}(t)\right] A_{3}(t) . \\
\frac{d}{d t} A_{4}(t) & =-\beta_{43} A_{3}(t) A_{4}(t) . \\
\frac{d}{d t} A_{5}(t) & =-\beta_{52} A_{2}(t) A_{5}(t) .
\end{aligned}
$$

Remark 5.1 From now on we will use the notation

$$
A_{i}^{0}:=A_{i}(0)=\int_{0}^{+\infty} u f_{i}^{0}(u) d u, \quad \text { and } \quad n_{i}^{0}:=n_{i}(0)=\int_{0}^{+\infty} f_{i}^{0}(u) d u,
$$

$\forall i=1, \ldots 5$.

In general we are not able to predict exactly the asymptotic behaviour given a set of initial data, except in the case where only the host and the immune system are present where we have the following result.

Proposition 5.1 For $f_{3}^{0}=f_{4}^{0}=f_{5}^{0}=0$, we define $n_{2}^{*}=\int e^{\beta_{21} u / \alpha_{21}} f_{2}^{0}(u) d u$ and we have that

i) If $\left(\alpha_{12}+\beta_{12}\right) n_{2}^{0}+\beta_{21} A_{1}^{0}<\left(\alpha_{12}+\beta_{12}\right) n_{2}^{*}$, then $A_{1} \rightarrow 0$ as $\rightarrow \infty$ and $A_{2}$ is uniformly bounded from below.

ii) If $\left(\alpha_{12}+\beta_{12}\right) n_{2}^{0}+\beta_{21} A_{1}^{0} \geq\left(\alpha_{12}+\beta_{12}\right) n_{2}^{*}$, then $A_{2} \rightarrow 0$ as $t \rightarrow \infty$.

Before presenting the partial results which we are able to obtain in the general case, we state the next lemma concerned with an estimate for the distribution $f_{2}$.

Lemma 5.2 Assume $f_{i}^{0}$ satisfy (5.1)-(5.3), $i=1, \ldots, 5$. Then we have for the activation $A_{2}(t)$ the following estimate

$$
A_{2}^{0} e^{\int_{0}^{t} A(s) d s} \leq A_{2}(t) \leq\left(\int u e^{C u} f_{2}^{0}(u) d u\right) \times e^{\int_{0}^{t} A(s) d s},
$$

where $C=\frac{\beta_{21}}{\alpha_{21}}+\frac{\beta_{21} \alpha_{25} A_{5}^{0}}{\alpha_{21} \alpha_{52} A_{2}^{0}}$ and $A(s)=\alpha_{25} A_{5}(s)-\alpha_{21} A_{1}(s)$.

The next two propositions will show that it is always possible to have a therapeutical action sufficiently high in order to have, asymptotically, the destruction of the tumour cells. 
Proposition 5.2 It is always possible to choose $A_{5}^{0}$ sufficiently high so that the activity of the immune system $A_{2}$ together with $A_{3}$ are bounded from below by a positive constant and $A_{1}$ converges toward 0 as time tends to infinity.

Concerning the first therapy, the result is a bit weaker as it reads:

Proposition 5.3 If $A_{5}^{0}=0$, and $\left(\alpha_{12}+\beta_{12}\right) n_{2}^{0}+\beta_{21} A_{1}^{0}>\left(\alpha_{12}+\beta_{12}\right) n_{2}^{*}$, it is possible to choose $A_{4}^{0}$ such that $A_{2}$ is bounded from below and both $A_{1}$ and $A_{3}$ converge toward zero as time tends to infinity.

Notice that this result is optimal in the sense that if $A_{5}^{0}=0$, and $2 n_{2}^{0}+A_{1}^{0}<2 n_{2}^{*}$, then for any $A_{4}^{0}$, it is $A_{2}$ which converges to 0 since we have convergence toward the asymptotic behaviour prescribed by Prop. 5.1. We do not know the precise condition if $A_{5}^{0}$ is non zero.

We stress the point that the previous two propositions are independent from each other. Let us now investigate the asymptotic behaviour in time of the solution.

We will use the following lemma.

Lemma 5.3 For the solutions to the initial value problem (4.1)-(4.6) given by Theorem 5.1 we have

$$
\begin{aligned}
& \int_{0}^{+\infty} A_{2}(t) A_{5}(t) d t<+\infty \\
& \int_{0}^{+\infty} A_{3}(t) A_{4}(t) d t<+\infty \\
& \int_{0}^{+\infty} A_{1}(t) A_{3}(t) d t<+\infty .
\end{aligned}
$$

From Eq. (5.5), $A_{3}(t)$ is decreasing, and hence

$$
\text { either } \quad A_{3} \rightarrow 0 \quad \text { or } \exists c>0 \text { s.t. } \quad A_{3}(t) \geq c, \forall t .
$$

First, we consider the asymptotic behaviour in the case $A_{4}^{0}=0$.

Theorem 5.2 Assume that $A_{5}^{0} \neq 0$ and $A_{4}^{0}=0$. Then as $t \rightarrow+\infty$, there are only the following two possibilities:

i) If $A_{3}(t)$ is bounded from below by a positive constant, then also $A_{2}(t)$ is bounded from below by a positive constant, $\int_{0}^{+\infty} A_{1}(t) d t<+\infty$ and $\int_{0}^{+\infty} A_{5}(t) d t<+\infty$;

ii) If $A_{3} \rightarrow 0$, then also $A_{2}(t) \rightarrow 0$ and $\int_{0}^{+\infty} A_{1}(t) d t=+\infty$.

We are not able to indicate all possible asymptotical features of the system, if $A_{5}^{0} \neq 0$ and $A_{4}^{0} \neq 0$, but we can still give some partial answers. In particular, property i) of Theorem (5.2) remains true.

Proposition 5.4 If $A_{3}(t)$ is bounded from below by a positive constant, then also $A_{2}(t)$ is bounded from below by a positive constant, $\int_{0}^{+\infty} A_{1}(t) d t<+\infty, \int_{0}^{+\infty} A_{4}(t) d t<+\infty$ and $\int_{0}^{+\infty} A_{5}(t) d t<+\infty$. 
An interesting biological interpretation can be given to the main results described in this section. First we observe that conditions (5.1)-(5.3) needed to prove the existence theorem are consistent with the biological requirements of cell populations. Specifically the decay to zero at infinity implied by conditions (5.1)-(5.3) can be regarded as natural, since the distribution functions $f_{i}$ must effectively decay to zero at infinity to have a bound to the initial density and activation as given by Eqs. (2.1) and (2.2). Exponential might nevertheless seem too demanding but we recall the result obtained in [5], which also applies here and which tells that, without some exponential decay, no solution exists even for a short time.

The theorem states in (5.4) the $L_{\infty}$ boundedness of the weighted distributions $f_{1}$ and $f_{2}$ and a bound of $f_{3}$ to $f_{5}$ with respect to the corresponding quantities at $t=0$. The theorem provides a useful background for simulations, while the results which are useful for a biological interpretation are stated in Propositions (5.1)-(5.4) and Theorem (5.2). In detail, Proposition (5.1) concerns the simplest case where only the tumour cells and the immune system are present and it indicates how the system chooses between the two asymptotic behaviours, already described in [5], in terms of the initial data and of the parameters of the model. We recall that in the general case studied in [5] we did not have such a result.

Propositions (5.2) and (5.3) show the efficiency of the treatment in both the cases of the two therapies considered, even if the analytical results obtained in the case of the angiogenesis control is a bit weaker. The two propositions state how in principle is always possible to reach the situation where the immune system wins and completely eliminates the tumour cells of the organism. As a consequence of the activation of the immune system or of the weakening of the feeding ability of the environmental cells, respectively, the activation of the tumour system evolves toward lower degrees of malignity. Unfortunately, these mathematical results do not always correspond to the reality, in the sense that the amount of the initial conditions of the treatments can be not realistic (if a high dose of treatment is dangerous for other reasons).

Theorem (5.2) shows the complete asymptotic analysis in the presence of the second therapy. Recalling that this is the therapy referring to the actions applied by proteins to activate the immune defense, the analysis corresponds to a well defined medical motivation related to the action of cytokine signals, [11] and [15]. The competition between the tumour cells and the immune system may end up with the regression of progressed cells, due to the action of the immune system, or with the blow up of progressed cells and inhibition of the immune system.

Finally, Proposition (5.4) gives only a partial answer concerning the asymptotic behaviour in the general case of the two therapies, showing a case where the destruction of the host is still possible. In that case, some more complicated asymptotic behaviours are probably possible, corresponding to chronical diseases for instance (with oscillations for the numbers of immune cells and agressive hosts). 


\section{$6 \quad$ Estimates and Proofs}

This section is devoted to the technical proofs of the qualitative properties of the solution to the Cauchy problem (4.1) - (4.6).

\subsection{Existence theorem 5.1}

The proof of the existence Theorem 5.1 is the same as that of Theorem 2.1 in [5]. We just list here the main steps of the proof:

- A priori estimates: any solution $f_{i}$, for $i=1, \ldots, 5$, to the system which is the weak limit of compactly supported solutions in $L_{t}^{\infty} L_{u}^{1}$, satisfies the estimates (5.4).

- Continuity in time: given $A_{i} \in L^{\infty}([0, T])$, for $i=1, \ldots, 5$, the weak solutions $f_{i}$, for $i=1, \ldots, 5$, to the system, satisfying the conditions (5.4), also belong to

$$
C\left([0, T], L^{1}((1+u) d u)\right), \quad \forall T>0 .
$$

\section{- Stability result:}

Let $f_{i}^{n}$ in $C\left([0, \infty), L^{1}((1+u) d u)\right)$, for $i=1, \ldots, 5$, be a sequence of solutions in the distributional sense. Assume that they satisfy the a priori estimates (5.4) uniformly in $n$. Then any weak limit $f_{i}$, for $i=1, \ldots, 5$, of converging subsequences also belongs to $C\left([0, \infty), L^{1}((1+u) d u)\right)$ and solves the system in distributional sense with initial data the weak limits of the initial data.

- Existence: we construct sequences of approximate solutions of our system, satisfying every a priori estimate. Taking weak limits, we obtain solutions for any initial data satisfying the hypotheses (5.1) - (5.3) of the theorem, on the interval $[0, T]$. Since the solutions satisfy the estimates (5.4) uniformly in $t$, we may extend indefinitely the time of existence.

\subsection{Proof of Proposition 5.1}

If $f_{3}^{0}=f_{4}^{0}=f_{5}^{0}=0$, they remain so at any latter time, which obviously simplifies the equations on $f_{1}$ and $f_{2}$. Multiplying Eq. (4.1) by $u$ and integrating we formally obtain in this case

$$
\frac{d}{d t} A_{1}(t)=-\left(\alpha_{12}+\beta_{12}\right) A_{1} A_{2}
$$

This formal computation can easily be made rigorous (see [5] for more details). Integrating Eq. (4.2) we get

$$
\frac{d}{d t} n_{2}(t)=\beta_{21} A_{1} A_{2}
$$

Then with these two relations, we conclude that

$$
\frac{d}{d t}\left(\left(\alpha_{12}+\beta_{12}\right) n_{2}+\beta_{21} A_{1}\right)=0 .
$$


We now recall the main result from [5] which states that either $A_{1}$ converges toward 0 , $A_{2}$ is bounded from below and $n_{2}$ is bounded away from $n_{2}^{*}$, or $A_{2}$ converges toward 0 and $n_{2}$ converges toward $n_{2}^{*}$.

The first case means that

$$
\lim _{t \rightarrow \infty}\left(\left(\alpha_{12}+\beta_{12}\right) n_{2}(t)+\beta_{21} A_{1}(t)\right)<\left(\alpha_{12}+\beta_{12}\right) n_{2}^{*},
$$

and the second that

$$
\lim _{t \rightarrow \infty}\left(\left(\alpha_{12}+\beta_{12}\right) n_{2}(t)+\beta_{21} A_{1}(t)\right) \geq\left(\alpha_{12}+\beta_{12}\right) n_{2}^{*} .
$$

These are exactly the two conditions given in Prop. 5.1.

\subsection{Proof of Lemma 5.1}

It is easy to see that the estimates given in the Lemma 5.1 are formally true. For a rigorous proof we refer to Lemma 5.5 in [5].

\subsection{Proof of Lemma 5.2}

Let us consider equation (4.2) for the distribution $f_{2}$ and the characteristics

$$
U(t, u)=u \times e^{\int_{0}^{t} A(s) d s}
$$

where $A(s)=\alpha_{25} A_{5}(s)-\alpha_{21} A_{1}(s)$. Then the equation for $f_{2}$ along the characteristics is given by

$$
\begin{aligned}
\frac{d}{d t}\left[f_{2}(t, U(t, u))\right] & \partial_{t} f_{2}(t, U(t, u))+\partial_{t} U(t, u) \partial_{u} f_{2}(t, U(t, u)) \\
& =\partial_{t} f_{2}(t, U(t, u))+A(t) U(t, u) \partial_{u} f_{2}(t, U(t, u)) \\
& =\left[\beta_{21} U(t, u) A_{1}(t)+A(t)\right] f_{2}(t, U(t, u))
\end{aligned}
$$

so that

$$
f_{2}(t, U(t, u))=f_{2}^{0}(u) \times e^{\int_{0}^{t}\left[\beta_{21} U(s, u) A_{1}(s)+A(s)\right] d s}
$$

and hence

$$
\begin{aligned}
& \int U f_{2}(t, U) d U \\
& =\int u \times e^{\int_{0}^{t} A(s) d s} \times f_{2}^{0}(u) \times e^{\int_{0}^{t}\left[\beta_{21} U(s, u) A_{1}(s)+A(s)\right] d s} \times e^{\int_{0}^{t} A(s) d s} d u .
\end{aligned}
$$

Consequently we get for $A_{2}$ the expression

$$
A_{2}(t)=\left(\int u f_{2}^{0}(u) \times e^{\int_{0}^{t}\left[\beta_{21} U(s, u) A_{1}(s)\right] d s} d u\right) \times e^{\int_{0}^{t} A(s) d s} .
$$


Let us now consider

$$
0 \leq \int_{0}^{t} \beta_{21} U(s, u) A_{1}(s) d s \beta_{21} u \times \int_{0}^{t} e^{\int_{0}^{s} A(\tau) d \tau} d s=: \beta_{21} u I(t)
$$

and

$$
\alpha_{21} I(t)=-\int_{0}^{t} e^{\int_{0}^{s} A(\tau) d \tau} \times A(s) d s+\alpha_{25} \int_{0}^{t} e^{\int_{0}^{s} A(\tau) d \tau} \times A_{5}(s) d s=: I_{1}(t)+I_{2}(t) .
$$

For the first integral we have

$$
I_{1}(t)=-\int_{0}^{t} \partial_{s}\left[e^{\int_{0}^{s} A(\tau) d \tau}\right] d s 1-e^{\int_{0}^{s} A(\tau) d \tau} \leq 1,
$$

while from (6.2) we deduce that

$$
A_{2}(t) \geq\left(\int u f_{2}^{0}(u) d u\right) \times e^{\int_{0}^{t} A(\tau) d \tau} A_{2}^{0} e^{\int_{0}^{t} A(\tau) d \tau} .
$$

Therefore

$$
0 \leq I_{2}(t) \leq \frac{\alpha_{25}}{A_{2}^{0}} \times \int_{0}^{t} A_{2}(s) A_{5}(s) d s \frac{\alpha_{25}}{\alpha_{52} A_{2}^{0}}\left(A_{5}^{0}-A_{5}(t)\right),
$$

where the last equality is obtain from Eq. (5.7). Finally,

$$
0 \leq I_{2}(t) \leq \frac{\alpha_{25} A_{5}^{0}}{\alpha_{52} A_{2}^{0}}
$$

and

$$
\alpha_{21} I(t) \leq 1+\frac{\alpha_{25} A_{5}^{0}}{\alpha_{52} A_{2}^{0}}
$$

so that we obtain

$$
A_{2}^{0} e^{\int_{0}^{t} A(s) d s} \leq A_{2}(t) \leq\left(\int u e^{C u} f_{2}^{0}(u) d u\right) \times e^{\int_{0}^{t} A(s) d s},
$$

where $C=\frac{\beta_{21}}{\alpha_{21}}+\frac{\beta_{21} \alpha_{25} A_{5}^{0}}{\alpha_{21} \alpha_{52} A_{2}^{0}}$, which proves Lemma 5.2.

\subsection{Proof of Proposition 5.2}

The proof of Prop. 5.2 will be carried out in two steps. The first step consists in showing that

$$
\forall K, \quad \exists A_{5}^{0} \quad \text { and } \quad \exists t_{0} \leq 1 \text { s.t. } \quad A_{2}\left(t_{0}\right)>K .
$$

Given any differentiable function $\lambda(t)$, let us define

$$
J(t)=\int e^{\lambda(t) u} f_{1}(t, u) d u .
$$


From Eq. (4.1) we have

$$
\frac{d}{d t} J(t) \leq \lambda^{\prime}(t) \int u e^{\lambda(t) u} f_{1}(t, u) d u+\left(\alpha_{13} \lambda(t)+\beta_{13}\right) A_{3}(t) \int u e^{\lambda(t) u} f_{1}(t, u) d u .
$$

Now we choose $\lambda$ s.t. $\lambda^{\prime}(t)=-\left(\alpha_{13} \lambda(t)+\beta_{13}\right) A_{3}^{0}$, and this implies

$$
\frac{d}{d t} J(t) \leq 0 \quad \text { and } \quad \lambda(t)=C e^{-\alpha_{13} A_{3}^{0} t}-\frac{\beta_{13}}{\alpha_{13}},
$$

where $C$ is an arbitrary constant. Now we take $C$ s.t. $\lambda(1)=1$ and consider the following estimate, $\forall t \leq 1$,

$$
\int e^{u} f_{1}(t, u) d u \leq \int e^{\lambda(t) u} f_{1}(t, u) d u=J(t) \leq J(0) \int e^{\left(C-\frac{\beta_{13}}{\alpha_{13}}\right) u} f_{1}^{0}(u) d u .
$$

Now

i) $A_{3}(t) \leq A_{3}^{0}, \quad \forall t \leq 1, \quad$ from $(5.5)$.

ii) $A_{2}(t) \geq A_{2}^{0} e^{\int_{0}^{t}\left(\alpha_{25} A_{5}(s)-\alpha_{21} A_{1}(s)\right) d s}$, from (5.8),

iii) $A_{1}(s) \leq \int e^{u} f_{1}(s, u) d u \leq \frac{1}{\alpha_{21}} \tilde{C}\left(A_{3}^{0}, f_{1}^{0}\right), \quad$ from (6.3),

and using property iii) in ii), we have

$$
A_{2}(t) \geq A_{2}^{0} e^{\int_{0}^{t} \alpha_{25} A_{5}(s) d s-\tilde{C} t} \geq A_{2}^{0} e^{-\tilde{C}} e^{\int_{0}^{t} \alpha_{25} A_{5}(s) d s}, \quad \forall t \leq 1 .
$$

Assume now that $\exists K$ s.t. $\forall A_{5}^{0}, A_{2}(t) \leq K, \forall t \leq 1$.

Recalling Eq. (5.7) we get

$$
A_{5}(t) \geq A_{5}^{0} e^{-\alpha_{52} K t}
$$

and

$$
A_{2}(t) \geq A_{2}^{0} e^{-\tilde{C}} e^{\frac{\alpha_{25} A_{5}^{0}}{\alpha_{52} K} \times\left[1-e^{-\alpha_{52} k}\right]} \longrightarrow+\infty, \quad \text { as } \quad A_{5}^{0} \rightarrow+\infty
$$

but this is a contradiction and the first step is proved.

Now, the second step consists in showing that we can choose $\alpha \leq 1$ and $K$ s.t. $A_{2}^{0} \geq K$ implies $A_{2}(t) \geq \alpha K$.

Let us choose $\alpha, K$ s.t. $A_{2}^{0} \geq K$ and $\alpha \leq 1$. Define $T$ the first time (if it exists) s.t. $A_{2}(t)=\alpha K$. Then, $\forall t \in[0, T], A_{2}(t) \geq \alpha K$.

By Eq. (4.1) we obtain

$$
\begin{aligned}
& \frac{d}{d t} \int e^{\lambda u} f_{1}(t, u) d u= \\
& \left(\lambda \alpha_{13} A_{3}(t)+\beta_{13} A_{3}(t)-\lambda \alpha_{12} A_{2}(t)\right) \\
& \times \int u e^{\lambda u} f_{1}(t, u) d u-\beta_{12} A_{2}(t) \int e^{\lambda u} f_{1}(t, u) d u
\end{aligned}
$$


If we choose $\lambda$ s.t.

$$
A_{3}^{0} \frac{\alpha_{13} \lambda+\beta_{13}}{\alpha_{12} \lambda} \leq \alpha K
$$

then we have

$$
\int e^{\lambda u} f_{1}(t, u) d u \leq e^{-\beta_{12} \alpha K t} \int e^{\lambda u} f_{1}^{0}(u) d u,
$$

and we use this estimate to deduce that

$$
A_{1}(t) \leq \frac{1}{\lambda} \int e^{\lambda u} f_{1}(t, u) d u \leq \frac{1}{\lambda} e^{-\beta_{12} \alpha K t} \int e^{\lambda u} f_{1}^{0}(u) d u .
$$

Using the previous computations and the relation

$$
\frac{d}{d t} A_{2}(t) \geq-\alpha_{21} A_{1}(t) A_{2}(t)
$$

that we obtain from Eq. (4.2), we have the following estimate for $A_{2}(t)$

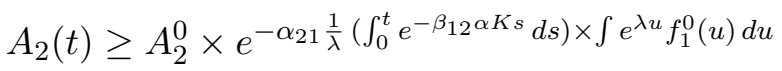

$$
\begin{aligned}
& K \times \exp \left(-\frac{\alpha_{21}}{\beta_{12} \lambda \alpha K} \times \int e^{\lambda u} f_{1}^{0}(u) d u\right)>\alpha K
\end{aligned}
$$

provided

$$
\exp \left(-\frac{\alpha_{21}}{\beta_{12} \lambda \alpha K} \times \int e^{\lambda u} f_{1}^{0}(u) d u\right)>\alpha .
$$

Summarizing, we can choose $\alpha, K$ s.t. (6.5) and (6.6) are satisfied, and in this case we get the contradiction that $A_{2}(t)>\alpha K$, and this proves the second step of the proof of Proposition 5.2.

\subsection{Proof of Proposition 5.3}

Take the equation for $A_{3}$ in Lemma 5.1, multiply it by $\beta_{43}$, and subtract it from the equation for $A_{4}$ in the same lemma, multiplied by $\alpha_{34}$, to obtain

$$
\frac{d}{d t}\left(\alpha_{34} A_{4}-\beta_{43} A_{3}\right)=\beta_{43}\left(\alpha_{31}+\beta_{31}\right) A_{1} A_{3} \geq 0 .
$$

As a consequence $\alpha_{34} A_{4}$ is larger than $K=\alpha_{34} A_{4}^{0}-\beta_{43} A_{3}^{0}$, and this constant as high as we want provided $A_{4}^{0}$ is high enough. Since

$$
\frac{d}{d t} A_{3} \leq-\alpha_{34} A_{4} A_{3},
$$

we deduce that

$$
A_{3}(t) \leq A_{3}^{0} e^{-K t}
$$

Now let us write down the characteristics for (4.1), which read

$$
\partial_{t} U(t, u)=\left(\alpha_{13} A_{3}-\alpha_{12} A_{2}\right) U(t, u), \quad U(0, u)=u .
$$


Thanks to (6.7), we have that

$$
U(t, u)=u \times e^{\int_{0}^{t}\left(\alpha_{13} A_{3}(s)-\alpha_{12} A_{2}(s)\right) d s} \leq u \times e^{\int_{0}^{t} \alpha_{13} A_{3}(s) d s} \leq \Gamma u
$$

with

$$
\Gamma=e^{\alpha_{13} A_{3}^{0} / K} .
$$

On the other hand

$$
\partial_{t}\left(f_{1}(t, U(t, u))\right) \leq\left(\beta_{31} A_{3}(t) U(t, u)+\alpha_{12} A_{2}-\alpha_{13} A_{3}\right) f_{1}(t, U(t, u)),
$$

and consequently

$$
f_{1}(t, u) \leq f_{1}^{0}\left(u e^{-\int_{0}^{t}\left(\alpha_{13} A_{3}(s)-\alpha_{12} A_{2}(s)\right) d s}\right) \times e^{\Lambda u} \times e^{-\int_{0}^{t}\left(\alpha_{13} A_{3}(s)-\alpha_{12} A_{2}(s)\right) d s},
$$

where $\Lambda$ is uniformly bounded in terms of $A_{4}^{0}$, provided this last quantity is bounded away enough from 0 since

$$
\Lambda=\beta_{13} \frac{\alpha_{13} A_{3}^{0}}{K} e^{\alpha_{13} A_{3}^{0} / K} .
$$

From the inequality on $f_{1}$ and (6.8), we may deduce that

$$
\begin{aligned}
\int\left(u+u^{2}\right) f_{1}(t, u) d u & \leq \int\left(U(t, u)+|U(t, u)|^{2}\right) e^{\Lambda U(t, u)} f^{0}(u) d u \\
& \leq 2 \Gamma^{2} \int\left(u+u^{2}\right) e^{\Lambda \Gamma u} f^{0}(u) \leq C
\end{aligned}
$$

where $C$ does not depend on $A_{4}^{0}$, provided $A_{4}^{0}$ is large enough (so that $K>1$ for instance).

To conclude the proof, we write that

$$
\frac{d}{d t}\left(\left(\alpha_{12}+\beta_{12}\right) n_{2}+\beta_{21} A_{1}\right)=\beta_{21}\left(\alpha_{13} A_{3} A_{1}+\beta_{13} A_{3} \int u^{2} f_{1}(t, u) d u\right) .
$$

Thanks to (6.7), we get

$$
\left(\alpha_{12}+\beta_{12}\right) n_{2}(t)+\beta_{21} A_{1}(t) \leq\left(\alpha_{12}+\beta_{21}\right) n_{2}^{0}+\beta_{12} A_{1}^{0}+\frac{\tilde{C}}{K},
$$

with $\tilde{C}$ uniformly bounded if $K>1$. Consequently we may choose $A_{4}^{0}$ large enough such that

$$
\limsup _{t \rightarrow \infty}\left(\left(\alpha_{12}+\beta_{12}\right) n_{2}(t)+\beta_{21} A_{1}(t)\right)<\left(\alpha_{12}+\beta_{12}\right) n_{2}^{*} .
$$

Applying again the main result of [5], as in the proof of Prop. 5.1, we know that $A_{1}$ (and $n_{1}$ ) converges toward zero whereas $A_{2}$ is bounded away from 0 , since the limit of $n_{2}$ is strictly less than $n_{2}^{*}$.

Before turning to another proof, we mention that if we had initially that

$$
\left(\alpha_{12}+\beta_{12}\right) n_{2}^{0}+\beta_{21} A_{1}^{0} \geq\left(\alpha_{12}+\beta_{12}\right) n_{2}^{*},
$$


then, no matter what are the values of $A_{3}$ and $A_{4}$, but provided $f_{5}=0$, we know that

$$
\frac{d}{d t}\left(\left(\alpha_{12}+\beta_{12}\right) n_{2}+\beta_{21} A_{1}\right) \geq 0 .
$$

We also know that the limit of $A_{1}$ is either non zero or that the limit of $n_{2}$ is $n_{2}^{*}$. This guarantees that $A_{2}$ converges toward 0 .

\subsection{Proof of Lemma 5.3}

From Eq. (5.7) we have

$$
\beta_{52} \int_{0}^{t} A_{2}(s) A_{5}(s) d s=A_{5}^{0}-A_{5}(t) \leq A_{5}^{0}, \quad \forall t
$$

and Eq. (5.9) is proved. In the same way, we derive Eq. (5.10) from Eq. (5.6). From Eq. (5.5) we obtain

$$
\left(\alpha_{31}+\beta_{31}\right) \int_{0}^{t} A_{1}(s) A_{3}(s) d s=A_{3}^{0}-A_{3}(t)-\alpha_{34} \int_{0}^{t} A_{3}(s) A_{4}(s) d s \leq \text { Const }, \quad \forall t,
$$

and this proves Eq. (5.11).

\subsection{Proof of Theorem 5.2}

As it was already observed, from Eq. (5.5) we have two possible cases: either $A_{3} \rightarrow 0$ or $\exists c>0$ s.t. $A_{3}(t) \geq c, \forall t$.

1) Let us start with the case $A_{3}$ bounded from below. From Eq. (5.5) we know that $\int_{0}^{+\infty} A_{1}(t) d t<+\infty$ and, as a consequence,

$$
\int_{0}^{t} A(s) d s \geq-\alpha_{21} \int_{0}^{+\infty} A_{1}(t) d t
$$

Using this inequality in the left hand side of Eq. (5.8), we have

$$
A_{2}(t) \geq A_{2}^{0} e^{-\alpha_{21} \int_{0}^{+\infty} A_{1}(t) d t}
$$

and this proves that $A_{2}$ is bounded from below. From Eq. (5.9), we have that $\int_{0}^{+\infty} A_{5}(t) d t<+\infty$.

2) Let now consider the case $A_{3} \rightarrow 0$. From Eq. (5.5) we know that

$$
\int_{0}^{+\infty} A_{1}(t) d t=+\infty .
$$

We analyze the following three subcases 
(a) $\int_{0}^{t} A(s) d s$ bounded from below, i.e. $\exists c \in \mathbb{R}$ s.t

$$
\int_{0}^{t} A(s) d s \geq c, \forall t \in \mathbb{R}^{+} .
$$

This implies that $A_{2}(t) \geq A_{2}^{0} e^{c}$, so from Eq. (5.7) we deduce the inequality $A_{5}(t) \leq A_{5}^{0} e^{-\alpha_{52} A_{2}^{0} e^{c} t}$ and as consequence $\int_{0}^{+\infty} A_{5}(s) d s<+\infty$.

Therefore, from Eq. (6.10), $\int_{0}^{t} A(s) d s \longrightarrow-\infty$, as $t \rightarrow+\infty$, but this is a contradiction, and $\int_{0}^{t} A(s) d s$ cannot be bounded from below.

(b) $\int_{0}^{t} A(s) d s$ converges to $-\infty$, as $t \rightarrow+\infty$ : from Eq (5.8) this directly implies

$$
A_{2}(t) \longrightarrow 0, \quad \text { as } t \rightarrow+\infty
$$

q.e.d.

(c) $\int_{0}^{t} A(s) d s$ be neither bounded from below nor converging to $-\infty$.

We may construct two sequences $\left\{t_{n}\right\}$ and $\left\{\tilde{t}_{n}\right\}$ s.t.

$$
t_{n} \leq \tilde{t}_{n} \leq t_{n+1}, \quad \int_{0}^{t_{n}} A(s) d s \leq-n \quad \text { and } \quad \int_{0}^{\tilde{t}_{n}} A(s) d s \geq c
$$

with $c$ constant.

We show that $A_{5}(t) \longrightarrow 0$, as $t \rightarrow+\infty$. Let $s_{n} \in\left[t_{n}, \tilde{t}_{n}\right]$ be the first time s.t.

$$
\int_{0}^{s_{n}} A(s) d s=c-1 \quad \text { and } \quad \int_{0}^{t} A(s) d s \geq c-1, \quad \forall t \in\left[s_{n}, \tilde{t}_{n}\right] .
$$

As

$$
\frac{d}{d t} \int_{0}^{t} A(s) d s=A(t) \leq \alpha_{25} A_{5}(t) \leq \alpha_{25} A_{5}^{0}
$$

we know that $\tilde{t}_{n}-s_{n} \geq \frac{1}{\alpha_{25} A_{5}^{0}}$. Moreover, $\forall t \in\left[s_{n}, \tilde{t}_{n}\right], A_{2}(t) \geq A_{2}^{0} e^{c-1}$ and so

$$
A_{5}\left(\tilde{t}_{n}\right) \leq A_{5}\left(s_{n}\right) \times e^{\alpha_{52} A_{2}^{0} e^{c-1}\left(\alpha_{25} A_{5}^{0}\right)^{-1}} .
$$

Consequently $A_{5}\left(\tilde{t}_{n}\right) \longrightarrow 0$ and this implies $A_{5}(t) \longrightarrow 0$, since $A_{5}$ is decreasing.

We now show that $A_{2}(t) \longrightarrow 0$, as $t \rightarrow+\infty$. From Eq (5.8) we know that:

$$
A_{2}(t) \leq\left(\int u e^{C u} f_{2}^{0}(u) d u\right) \times e^{\int_{0}^{t} A(s) d s} \leq K e^{\alpha_{25} \int_{0}^{t} A(s) d s} .
$$

Given any $t_{1} \leq t_{2}$, we have

$$
A_{2}\left(t_{1}\right) \geq A_{2}^{0} e^{\int_{0}^{t_{1}} A(s) d s} \quad \text { and } \quad A_{2}\left(t_{2}\right) \leq K e^{\int_{0}^{t_{2}} A(s) d s},
$$


SO

$$
\begin{aligned}
A_{2}\left(t_{2}\right) & \leq \frac{K}{A_{2}^{0}} A_{2}\left(t_{1}\right) e^{\int_{t_{1}}^{t_{2}} A(s) d s} \\
& \leq \tilde{K} A_{2}\left(t_{1}\right) \exp \left(\alpha_{25} A_{5}\left(t_{1}\right) \int_{t_{1}}^{t_{2}} e^{-\alpha_{52} \int_{t_{1}}^{s} A_{2}(\tau) d \tau} d s\right)
\end{aligned}
$$

Now we prove the following inequality

$$
A_{2}\left(t_{2}\right) \leq \tilde{K} A_{2}\left(t_{1}\right) \exp \left(\frac{\alpha_{25} A_{5}\left(t_{1}\right)}{\alpha_{52} A_{2}\left(t_{1}\right)}\right)
$$

for $t_{1}$ fixed and $\forall t \geq t_{1}$. If it is not true, then $\exists t_{2}$ with

$$
A_{2}\left(t_{2}\right)>\tilde{K} A_{2}\left(t_{1}\right) \exp \left(\frac{\alpha_{25} A_{5}\left(t_{1}\right)}{\alpha_{52} A_{2}\left(t_{1}\right)}\right) .
$$

Define $t_{0}$ as the largest time $t \leq t_{2}$ with $A_{2}(t)=A_{2}\left(t_{1}\right)$.

Clearly $t_{1} \leq t_{0} \leq t_{2}$ and $\forall t \in\left[t_{0}, t_{2}\right]: A_{2}(t) \geq A_{2}\left(t_{1}\right)$. Therefore

$$
\begin{aligned}
A_{2}\left(t_{2}\right) & \leq \tilde{K} A_{2}\left(t_{0}\right) \exp \left(\alpha_{25} A_{5}\left(t_{0}\right) \int_{t_{0}}^{t_{2}} e^{-\alpha_{52}\left(s-t_{0}\right) A_{2}\left(t_{1}\right)} d s\right) \\
& \leq \tilde{K} A_{2}\left(t_{1}\right) \exp \left(\frac{\alpha_{25} A_{5}\left(t_{0}\right)}{\alpha_{52} A_{2}\left(t_{1}\right)}\right) \\
& \leq \tilde{K} A_{2}\left(t_{1}\right) \exp \left(\frac{\alpha_{25} A_{5}\left(t_{1}\right)}{\alpha_{52} A_{2}\left(t_{1}\right)}\right),
\end{aligned}
$$

as $A_{5}$ is decreasing, but this is a contradiction, and Eq. (6.11) is proved. Now we know that $A_{5} \longrightarrow 0$ and take $t_{n} \leq \tilde{t}_{n} \leq t_{n+1}$ such that $A_{2}\left(t_{n}\right) \longrightarrow 0$ and $A_{2}\left(\tilde{t}_{n}\right)$ is bounded from below.

We can find a sequence $r_{n}$ s.t. $A_{2}\left(r_{n}\right) \longrightarrow 0$ and $\frac{A_{5}\left(r_{n}\right)}{A_{2}\left(r_{n}\right)} \leq \frac{\alpha_{52}}{\alpha_{25}}$.

Then $\forall t \geq r_{n}$, from Eq. (6.11), $A_{2}(t) \leq \tilde{K} A_{2}\left(r_{n}\right) e$ and $A_{2} \longrightarrow 0$, but this contradicts the fact that $A_{2}\left(\tilde{t}_{n}\right)$ is bounded from below. So it is proved that $A_{2} \longrightarrow 0$.

\subsection{Proof of Proposition 5.4}

We are in the case $\exists c>0$ s.t. $A_{3}(t) \geq c, \forall t$. From Eq. (5.6) we have

$$
A_{4}(t) \leq A_{4}^{0} e^{-\beta_{43} c t}, \quad \text { hence } \int_{0}^{+\infty} A_{4}(t) d t<+\infty,
$$

and because of Eq. (5.5) this implies

$$
\int_{0}^{+\infty} A_{1}(t) d t<+\infty
$$


We also have, by definition,

$$
\int_{0}^{t} A(t) d t \geq-\alpha_{21} \int_{0}^{t} A_{1}(s) d s \geq-\alpha_{21} \int_{0}^{+\infty} A_{1}(s) d s,
$$

and from Eq. (5.8)

$$
A_{2}(t) \geq A_{2}^{0} e^{-\alpha_{21} \int_{0}^{+\infty} A_{1}(s) d s},
$$

so $A_{2}$ is bounded from below. Finally, from Eq. (5.9) we have $\int_{0}^{+\infty} A_{5}(t) d t<+\infty$ and this concludes the proof.

\section{References}

[1] Arlotti L., Bellomo N. and De Angelis E., Generalized Kinetic (Boltzmann) Models: Mathematical Structures and Applications, Math. Models Methods Appl. Sci., 12, 567-591, (2002).

[2] Bellomo N., Bellouquid A., and Delitala M., Mathematical topics on the modeling complex multicellular systems and tumour immune cells competition, Math. Models Methods Appl. Sci., 14, 1683-1733, (2004).

[3] Bellomo N., De Angelis E., Preziosi L., Multiscale Modeling and Mathematical Problems Related to Tumour Evolution and Medical Therapy, Journal of Theor. Med., 5 , 111-136, (2003).

[4] De Angelis E. and Mesin L., Modelling of the Immune Response: Conceptual Frameworks and Applications, Math. Models Methods Appl. Sci., 11, 1609-1630, (2001).

[5] De Angelis E. and Jabin P.E., Qualitative analysis of a mean field model of tumour-immune system competition, Math. Models Methods. Appl. Sci., 13, 187206, (2003).

[6] Delves P. J. and Roitt Y. M., The immune system, Advances in Immunology, 343, 37-49, (2000).

[7] Derbel L., Analysis of a new model for tumor-immune system competition including long time scale effects, Math. Models Methods. Appl. Sci., 14, 1657-1681, (2004).

[8] Dredge K. et al., Adjuvant and the promo immunotherapy, Cancer Immunol. Immunother., 51, 521-531, (2002).

[9] Folkman J., Angiogenesis, in Harrison's Principle of International Medicine, Braunwald E. et al. Eds, 517-530, (McGraw-Hill, New York), (2001).

[10] Folkman J. and Kerbel K., Clinical translation of angiogenesis inhibitors, Nature Reviews Cancer, 2, 727-739, (2002). 
[11] Forni G. et al., Combined allogeneic tumour cell vaccination and systemic interleukin 12 prevents mammary carcinogenesis in HER-2/neu transgenic mice, $J$. Exp. Medicine, 194, 1195-1206, (2001).

[12] Greller L., Tobin F., and Poste G., Tumor hetereogenity and progression: conceptual foundation for modeling, Invasion and Metastasis, 16, 177-208, (1996).

[13] Hartwell H.L., Hopfield J.J., Leibner S., and Murray A.W., From molecular to modular cell biology, Nature, 402, c47-c52, (1999).

[14] Jain R.K., Molecular regulation of vessel maturation, Nature Medicine, 9, 685693, (2003).

[15] Lollini P.L. and Forni G., Antitumour vaccines: is it possibe to prevent a tumour ?, Cancer Immunol. Immunother., 51, 409-416, (2002).

[16] Nani F. and Freedman H.I., A mathematical model of cancer treatment by immunotherapy, Math. Biosci., 163, 159-199, (2000). 